\title{
A Characterization of Calculus I Final Exams in U.S. Colleges and Universities
}

\author{
Michael A. Tallman ${ }^{1,2}$ - Marilyn P. Carlson ${ }^{2}$. \\ David M. Bressoud ${ }^{3}$ - Michael Pearson ${ }^{4}$
}

Published online: 14 January 2016

C Springer International Publishing Switzerland 2016

\begin{abstract}
In this study, we developed a three-dimensional framework to characterize post-secondary Calculus I final exams. Our Exam Characterization Framework (ECF) classifies individual exam items according to the cognitive demand required to answer the item, the representation of both the task statement and the solution, and the item's format. Our results from using the ECF to code 150 post-secondary Calculus I final exams from across the United States revealed that the exams generally require low levels of cognitive demand, seldom contain problems stated in a real-world context, rarely elicit explanation, and do not require students to demonstrate or apply their understanding of the course's central ideas. We compared the results from analyzing individual instructor's exams with survey data of their beliefs about the conceptual orientation of their exams. Our analysis revealed inconsistencies between our characterization of Calculus I final exams and instructors' perceptions of their final exams relative to their conceptual focus and the extent to which the exam items ask students to explain their thinking. We also compared the characteristics of our sample of final
\end{abstract}

Michael A. Tallman

michael.tallman@okstate.edu

Marilyn P. Carlson

marilyn.carlson@asu.edu

David M. Bressoud

bressoud@macalester.edu

Michael Pearson

pearson@maa.org

1 Department of Mathematics, Oklahoma State University, 401 MSCS, Stillwater, OK 74074, USA

2 School of Mathematical and Statistical Sciences, Arizona State University, P.O. 87804, Tempe, AZ 85287, USA

3 Department of Mathematics, Statistics, and Computer Science, Macalester College, 1600 Grand Avenue, Saint Paul, MN 55105, USA

4 Mathematical Association of America, 1529 Eighteenth Street Northwest, Washington, DC 20036, USA 
exams with post-secondary Calculus I final exams administered in 1986/87. We found that Calculus I final exams in U.S. colleges and universities have changed very little in the past 25 years with respect to the percentage of exam items that require students to apply their understanding of foundational concepts, which suggest that the calculus reform movement of the late 1980s has had little effect on what is being assessed in current Calculus I courses in U.S. postsecondary institutions.

Keywords Calculus · Assessment $\cdot$ Mathematical reasoning $\cdot$ University level mathematics

\section{Introduction}

Course exams are among the most revealing written artifacts of the mathematical skills and understandings instructors want their students to acquire in a mathematics course. A course exam provides information about an instructor's expectations for students' level of computational fluency, their depth of understanding specific concepts, and the degree to which students are expected to make connections among the course's central ideas.

Our review of the literature related to Calculus I assessment revealed that little is known about the content of Calculus I exams administered in colleges and universities in the United States. Several studies have characterized items on mathematics exams and in textbooks according to their conceptual focus (Bergqvist 2007; Boesen et al. 2006; Gierl 1997; Li 2000; Lithner 2000, 2003, 2004; Mesa et al. 2012; Palm et al. 2006), while others have examined the format of exam questions (Senk et al. 1997), and the degree to which students can solve problems by imitating procedures (Bergqvist 2007; Lithner 2000, 2004). These approaches, however, characterize a small sample of exam and textbook items and therefore provide a limited snapshot of the mathematics valued by instructors or curriculum developers. The present study provides one response to this gap in the literature by characterizing a large number of final exams from first-semester calculus courses at a variety of post-secondary U.S. institutions. The four research questions that guided this study were:

1. What are the characteristics of post-secondary Calculus I final exams in the United States?

2. How do instructors' perceptions of their exams compare with our characterizations of them?

3. How is an exam item's representation and format related to the level of cognitive demand the item elicits?

4. How do the characteristics of our sample of modern post-secondary Calculus I final exams compare with those from 1986/87?

We begin this paper by outlining our study's context. We then chronicle the development of our Exam Characterization Framework (ECF), including details on how the existing literature informed its development and how iterative coding led to its refinement. Next, we describe the three strands of the ECF and provide examples of coded items to clarify potentially problematic interpretations. We then present the 
results from coding a random sample of 150 post-secondary Calculus I final exams, collectively containing 3735 items. This is followed by a presentation of results from comparing instructors' perceptions of their exams with our characterization of them. We conclude by comparing our characterization of the sample of 150 exams with the cognitive demand of a sample of Calculus I final exams from 1986/1987 administered at 13 different colleges and universities in the United States (Steen 1988).

\section{Context of Study}

This study is a part of a larger initiative by the Mathematical Association of America to determine the characteristics of successful programs in college calculus. As part of a larger data corpus, faculty from 253 universities electronically submitted instructor surveys and Calculus I final exams from the 2010/2011 academic year. Of these 253 exams, we randomly selected 150 for use in this study. We decided to analyze a random sample of 150 exams because we expected our results to stabilize after having coded such a large subsample. As we analyzed the exams, we kept track of the percentage of exam items classified within each category of the ECF and noticed that these percentages became stable after having coded about 100 exams. We therefore did not see any need to code the entire sample of 253 exams. Additionally, because we randomly selected 150 exams from a rather arbitrary sample size of 253, we found no statistically convincing reason to analyze the entire sample.

At the time the instructors provided data for the larger data corpus, $48 \%$ were tenured or tenure-track faculty, $28 \%$ were other full-time faculty, $9 \%$ were part-time faculty, and $15 \%$ were graduate students. Moreover, of the 150 exams we randomly selected, $61.9 \%$ were administered at national universities, $22.7 \%$ at regional universities, $9.4 \%$ at community colleges, $4.6 \%$ at national liberal arts colleges, and $1.4 \%$ at regional colleges. ${ }^{1}$

\section{Literature that Informed Our Development of the Exam Characterization Framework}

A major product of this study is the Exam Characterization Framework (ECF), which provides a means by which one can efficiently code mathematics exam items to achieve an objective characterization of the exam itself. It was our goal to locate or create a framework to characterize items relative to their conceptual versus procedural orientation, the representational context in which the items are presented (e.g., word problems, formulas, graphs) and the format of the items. In the first stage of developing our framework, we examined other frameworks (e.g., Li 2000; Lithner 2004; Mesa 2010; Mesa et al. 2012; Smith et al. 1996) that have been used to characterize items on exams and in textbooks. While our review of the literature allowed us to identify issues that

\footnotetext{
${ }^{1}$ National universities are those that offer a full range of undergraduate majors as well as a host of master's and doctoral degrees. Regional universities offer a full range of undergraduate programs, some master's programs, and few doctoral programs. National Liberal Arts Colleges are schools that emphasize undergraduate education and award at least half of their degrees in the liberal arts fields of study. Regional colleges are institutions focusing on undergraduate education, awarding less than half of their degrees in liberal arts fields of study. We used the university classifications of U.S. News \& World Report (www.usnews.com/education).
} 
were important for us to consider, we did not locate a framework that was suited for our purpose. The ECF emerged from 12 cycles of our coding exam items, refining our characterization of ECF constructs, and recoding items until we were satisfied that the constructs that comprise the ECF were accurately characterizing the exam items in our random sample and effectively distinguishing qualitatively distinct items.

In the remainder of this section, we summarize the various frameworks that informed our design of the ECF. This literature serves as a backdrop against which we present our framework in the following section.

\section{Item Coding Frameworks}

Li (2000) characterized items focused on the addition and subtraction of integers in American and Chinese mathematics textbooks. Through her examination of these textbook items, Li identified three dimensions of problem requirements: (a) mathematical feature, (b) contextual feature, and (c) performance requirements. The mathematical feature strand refers to the number of computational procedures needed to solve a problem. Li coded items as either single computation procedure required (S) or multiple computation procedures required $(\mathrm{M})$. The contextual feature dimension of her framework refers to the nature of contextual information contained in a problem statement, such as whether the problem statement was situated in a purely mathematical context in numerical or word form (PM) or illustrative context with pictorial representation or story (IC) (Li 2000, p. 237). Li further partitions the performance requirement dimension of her framework into two categories: (a) response type, and (b) cognitive requirement. Response type refers to the contextual feature of the solution that a task elicits whereas cognitive requirement refers to the mental act in which one engages while solving a task.

Lithner (2004) developed a cognitive framework to classify calculus textbook items according to various strategies that could be employed to complete the item. His interest in coding textbook items was based on his belief that most items in calculus textbooks could be completed without considering the intrinsic mathematical properties of the item. Lithner's framework includes six reasoning practices that one might employ when solving calculus textbook exercises, including reasoning based on identification of similarities, reasoning based on what seems to be true based on past experiences, and repeated algorithmic reasoning.

Smith et al. (1996) propose a taxonomy for classifying the cognitive demand of assessment tasks that evolved from the six intellectual behaviors in the cognitive domain of Bloom's taxonomy (Bloom et al. 1956). ${ }^{2}$ Smith et al. adapted Bloom's initial taxonomy for use in a mathematical context and proposed the taxonomy categories: (1) factual knowledge, (2) comprehension, (3) routine use of procedures, (4) information transfer, (5) application in new situations, (6) justifying and interpreting, (7) implications, conjectures, and comparisons, and (8) evaluation. The primary purpose of their taxonomy was to assist instructors in writing exam items.

Anderson and Krathwohl (2001) developed a revision of Bloom's taxonomy to reflect developments in cognitive psychology occuring after the publication of Bloom's

\footnotetext{
${ }^{2}$ These six intellectual behaviors are: knowledge, comprehension, application, analysis, synthesis, and evaluation.
} 
original taxonomy (Bloom et al. 1956). They also modified the categories in the cognitive domain of Bloom's original taxonomy to make the revised taxonomy more suited for classifying teaching goals (e.g., defining learning objectives).

Mesa et al. (2012) analyzed the characteristics of examples in college algebra textbooks along the four dimensions proposed by Charalambous et al. (2010): (1) cognitive demand, (2) the expected response, (3) the use of representations, and (4) the strategies available for verifying the correctness and appropriateness of the solution. The cognitive demand dimension consists of the categories: memorization, procedures without connections, procedures with connections, and doing mathematics. A student can solve tasks that require the cognitive behavior of memorizing by simply recalling information from memory without enacting a procedure. Tasks that require students to employ procedures without connections can be solved by applying rehearsed procedures without attending to connections to other mathematical ideas or real world contexts. Tasks that require students to employ procedures with connections prompt them to apply a procedure in which they draw connections to other mathematical ideas or real world contexts. Finally, tasks that expect students to do mathematics require them to investigate fundamental mathematics concepts that promote the generation of new knowledge.

The expected response dimension of Mesa et al.'s analytical framework refers to the solution that an example provides by identifying whether the solution offers only an answer, gives an answer with an explanation or justification of the process undertaken to arrive at that answer, or contains both an answer and a mathematical sentence. Moreover, Mesa et al. identified the representation of the statement of the example and the solution provided according to five representation categories: symbols, tables, graphs, numbers, and verbalizations.

\section{Development of the Exam Characterization Framework}

Our development of the Exam Characterization Framework (ECF) began with us familiarizing ourselves with the random sample of 150 Calculus I final exams. In doing so we identified items that we conjectured might be difficult to characterize relative to the level of cognition required for a correct response. Since we were most interested in characterizing the cognitive demand of exam items, our initial coding involved identifying the level of cognition required to solve an item. We chose to call this dimension item orientation.

Our initial attempt to apply the three cognitive requirements in Li's (2000) framework (mathematical feature, contextual feature, and performance requirements) in our coding revealed that these constructs were too coarsely defined for our purpose, and thus resulted in qualitative variation of items within each category with respect to the cognitive demand needed to respond to them. When attempting to use the various reasoning practices defined in Lithner's (2004) framework, we found that without knowledge of students' prior experiences, the boundaries of each classification lacked the specificity required to code items with a high degree of reliability. However, we did adopt Lithner's approach to classifying items based on the cognitive demand that was necessary for providing a correct response. Attending to the cognitive demand required by a task was necessary to achieve a reliable characterization of exam items since we did not have knowledge of students' experiences in their calculus courses. 
We became aware of Mesa et al.'s framework after having developed a complete draft of the ECF, but decided to consider her constructs for the purpose of refining our framework, especially since her dimensions had some similarities with those in the ECF. We attempted to code select exam items using the cognitive demand dimension of Mesa et al.'s (2012) framework, but found their characterization of the cognitive demand of exam items more related to desired understandings instead of the understandings that a task requires. Moreover, we had difficulty agreeing on precisely what constitutes a connection for an exam item. While discerning whether or not exam items evoked connections, we were unable to reliably categorize items within the "procedures without connections" and "procedures with connections" categories of the cognitive demand dimension of Mesa et al.'s framework.

The categories of Smith et al.'s (1996) framework, which evolved from Bloom's origional taxonomy (Bloom et al. 1956), appealed to us, but because they were designed to assist instructors in writing exam items, their categories lacked the hierarchical structure that a diagnostic taxonomy typically requires. Therefore, Anderson and Krathwohl's (2001) modification of the six intellectual behaviors in the cognitive domain of Bloom's taxonomy was the most helpful for informing our approach to coding the cognitive demand of exam items. Anderson and Krathwohl (2001) developed their revision of Bloom's taxonomy to reflect developments in cognitive psychology occuring after the publication of Bloom's original taxonomy, and to make the taxonomy more suited to the purposes of classroom teaching (e.g., defining learning objectives). They characterize the levels of their taxonomy using the verbs: remembering, understanding, applying, analyzing, evaluating, and creating.

Since we were evaluating tasks rather than actual behaviors, our definitions needed to focus exlusively on the nature of the exam items, leading us to consider the categories: remember, understand, apply, analyze, evaluate, and create. Coding the cognitive demand of calculus exam items using these constructs revealed that some items (e.g., determine the derivative of $f$ ) required students to use procedural skills while requiring no understanding of the concept(s) on which the skills are based. This required a different cognitive behavior than simply recalling information, and led to our introducing the construct recall and apply procedure to Anderson and Krathwohl's levels. Similarly, our preliminary coding revealed that one could demonstrate an understanding of a concept without applying the understanding to achieve a goal or solve a problem, resulting in our introducing the level apply understanding.

To classify exam items relative to the cognitive demand they elicit, we have adapted the six intellectual behaviors in the conceptual knowledge dimension of Anderson and Krathwohl's modified Bloom's taxonomy to (1) reflect the variety of cognitive behaviors elicited by mathematics tasks, and (2) accurately characterize the cognitive demand of mathematics tasks in the absence of information about students' prior knowledge for whom the tasks were intended. We describe these constructs, and our use of them, in more detail in the following section.

In the process of coding items, we found it relatively straightforward to specify the representation in which mathematics items are presented. Consistent with Li (2000) and Mesa et al. (2012), we also found it useful to code both the representation of the problem statement and the representation of the solution. We noted a representation type in the solution only if it was necessary to solve the problem or complete the task. 
The mathematical feature dimension of Li's framework led us to consider the coding dimension item density. We defined a dense item as one that necessitates the incorporation of multiple concepts, procedures, and skills. However, since calculus tasks are often subject to being solved in a variety of ways, we were unable to specify how many procedures and concepts were needed to solve a specific calculus problem. Moreover, the grain-size of "procedure" has a wide range of interpretations, thus making coding problematic for more complicated tasks. We thus determined that our attempts to code items according to the number of procedures and concepts that are needed to solve a problem was less important than the overall cognitive demand of the task. As a result, we abandoned the item density dimension early in our framework development.

We also considered the fourth dimension of Mesa et al.'s framework that identifies the strategies needed for students to employ control (i.e., verify the correctness and appropriateness of a solution) during the process of completing a task. Our efforts to code for control led us to recognize that control is exercised idiosyncratically since it is initiated by a student's assessment of the validity and appropriateness of their own work. For example, one student might take the time to reflect on the reasonableness of computations when working a multi-step applied problem, while another student might continue with a sequence of memorized steps and pay no attention to computations that produce unreasonable results. There are, however, instances in which students are more likely to exhibit control. Two such instances are when a question prompts students to provide either an explanation or justification for their work. Mesa et al. refer to such actions as further elaboration. We conjecture that the act of providing an explanation or justification might require one to reflect upon and assess the correctness of his or her work. However, if the teacher had the student practice justifying a response or solution, in homework or during class, it is possible that the student did not exercise control when responding to the exam item. Therefore, instead of coding for a mental process that a student might or might not exercise when responding to an item, we decided to not code for control but rather to add a dimension in the item format category for noting instances in which exam items require students to explain or justify their response.

\section{Exam Characterization Framework}

The Exam Characterization Framework characterizes exam items according to three distinct item attributes: (a) item orientation, (b) item representation, and (c) item format. The first two authors independently coded five Calculus I final exams while making note of each exam item's characteristics. We then met to compare and refine our characterizations so as to ensure that our meanings were consistent. After 12 cycles of coding and meeting to compare and refine our characterizations, we emerged with a stable framework that included categories that we found to be most useful for characterizing Calculus I final exam items. Two other coders coded ten randomly selected exams for the purpose of establishing the readability of our construct descriptions. After we refined the framework, the lead author independently coded all 150 exams in the sample.

The item orientation dimension of the framework includes the following seven categories of intellectual behaviors needed to respond to an exam item: remember, recall and apply procedure, understand, apply understanding, analyze, evaluate, and 
create. When coding items for their representation type, we coded representations present in the question or problem statement. We also coded for the representations required when responding to a question or working a problem. This dimension of our framework includes the categories: applied/modeling, symbolic, tabular, graphical, definition/theorem, proof, example/counterexample, and explanation. The third dimension of our framework is item format, with the categories multiple choice, short answer, and broad open-ended. When coding the format of the item, we also noted whether the item required students to provide a justification or explanation.

\section{Item Orientation}

The six classifications of the item orientation taxonomy are hierarchical, as is Bloom's taxonomy and Anderson and Krathwohl's (2001) modification of Bloom's taxonomy. The lowest level requires students to remember information and the highest level requires students to make connections (see Table 1). When coding items using the item orientation categories, we classified an item at a specific level only when this cognitive behavior was necessary for responding to the item. Even if an item was designed to assess a student's understanding of an idea, if a student is able to solve the problem by applying a memorized procedure, then we classified the item as "recall and apply a procedure." As emphasized above, since we had no information about students' experiences in their calculus courses, we attended only to the highest-level cognitive behavior that a task required. We also made a distinction between items that require students to understand an idea and those that require students to apply their understanding of an idea or concept (e.g., derivative) to solve the problem. The intellectual behaviors in the item orientation taxonomy of the ECF are described in more detail in Table 1.

Note that item orientation is not the same as item difficulty. The former takes as the unit of analysis the type of cognition an exam item elicits while the latter focuses on an item's complexity. A procedural item might be very complex (e.g., differentiating a complicated function) while a item that requires understanding might be relatively straightforward (e.g., explain what the output value of a point on the derivative function represents).

\section{Characterizing the Item Orientation Dimensions}

This subsection presents examples of Calculus I items that require the various cognitive behaviors within the item orientation taxonomy of the ECF. We provide examples from the first five categories of the item orientation taxonomy only, since none of the Calculus I final exam items we coded met the criteria for eliciting the cognitive behaviors "evaluate" or "create." We chose these examples to illustrate some of the more difficult interpretations we made during coding. These descriptions should also help to clarify our taxonomy categories.

Exam items that elicit the cognitive behavior of remembering prompt the student to recall factual information, but do not require the student to apply a procedure or demonstrate understanding. As one example, a test question that we classified as "remember" prompted students to recall a part of the statement of the Mean Value Theorem (Fig. 1). Successfully answering the question in Fig. 1 does not require 
Table 1 Adaptation of the six intellectual behaviors from Anderson and Krathwohl (2001)

\begin{tabular}{ll}
$\begin{array}{l}\text { Cognitive } \\
\text { behavior }\end{array}$ & Description \\
\hline Remember & $\begin{array}{c}\text { Students are prompted to retrieve knowledge from long-term memory (e.g., write the } \\
\text { definition of the derivative). }\end{array}$ \\
$\begin{array}{c}\text { Recall and apply } \\
\text { procedure }\end{array}$ & $\begin{array}{c}\text { Students must recognize what procedures to recall when directly prompted to do so in the } \\
\text { context of a problem (e.g., find the derivative/limit/antiderivative of } f \text { ). }\end{array}$ \\
$\begin{array}{l}\text { Understand } \\
\text { Students are prompted to make interpretations, provide explanations, make comparisons or } \\
\text { make inferences that require an understanding of a mathematics concept. }\end{array}$ \\
$\begin{array}{c}\text { Apply } \\
\text { understanding }\end{array}$ \\
$\begin{array}{c}\text { Students must recognize when to apply a concept when responding to a question or when } \\
\text { working a problem. To recognize the need to apply, execute or implement a concept in } \\
\text { the context of working a problem requires an understanding of the concept. }\end{array}$ \\
$\begin{array}{c}\text { Students are prompted to break material into constituent parts and determine how parts relate } \\
\text { to one another and to an overall structure or purpose. Differentiating, organizing, and } \\
\text { attributing are characteristic cognitive processes at this level (Krathwohl 2002, p. 215). }\end{array}$ \\
$\begin{array}{c}\text { Students are prompted to make judgments based on criteria and standards. Checking and } \\
\text { Evaluate } \\
\text { critiquing are characteristic cognitive processes at this level (Krathwohl 2002, p. 215). }\end{array}$ \\
$\begin{array}{c}\text { Students are prompted to put elements together to form a coherent or functional whole; } \\
\text { Create }\end{array}$ \\
$\begin{array}{l}\text { reorganize elements into a new pattern or structure. Generating, planning, and producing } \\
\text { are characteristic cognitive processes at this level (Krathwohl 2002, p. 215). }\end{array}$
\end{tabular}

students to demonstrate their understanding of the Mean Value Theorem, nor apply it to solve a problem. Simply recalling the statement of the theorem itself is sufficient.

Test items that require students to recall and apply a procedure typically contain verbal or symbolic cues that prompt students to use a definition, rule, theorem, algorithm, or procedure to determine an answer. These items commonly appear in a section of a test where students are directed to differentiate or integrate a function, or evaluate a limit. Such test items require students to apply differentiation rules, integration techniques, or limit properties in an algebraic context but do not require students to understand the rationale for their actions. For the purpose of contrasting the "recall and apply procedure" category with the "remember" category, consider the task in Fig. 2, which requires the use of the Mean Value Theorem.

The task (Fig. 2) prompts students to employ a procedure using the conclusion of the Mean Value Theorem without requiring them to demonstrate an understanding of the Mean Value Theorem. That is, the task does not require the student to recognize that the Mean Value Theorem ensures that, for a function $f$ that is continuous on the closed

Let $f$ be continuous on the closed interval $[a, b]$ and differentiable on the open interval $(a, b)$.

Then there exists some $c$ in $(a, b)$ such that
a. $f(c)=0$
b. $f^{\prime}(c)=0$
c. $\frac{f(b)-f(a)}{b-a}=f^{\prime}(c)$
d. $\frac{f^{\prime}(b)-f^{\prime}(a)}{b-a}=f(c)$

Fig. 1 Sample item requiring the cognitive behavior of remembering 
Show that the function $f(x)=x^{2}$ satisfies the hypotheses of the Mean Value Theorem on the interval $[0,4]$ and find a solution $c$ to the equation,

$$
\frac{f(b)-f(a)}{b-a}=f^{\prime}(c)
$$

on this interval.

Fig. 2 Sample item requiring the cognitive behavior of recalling and applying a procedure

interval $[a, b]$ and differentiable on the open interval $(a, b)$, there exists a point $c$ on $(a$, $b$ ) such that the average rate of change of $f$ over $[a, b]$ is equal to the instantaneous rate of change (or derivative) of $f$ at $c$. Moreover, the task does not require the student to justify why the statement of the Mean Value Theorem is true, nor does it require students to interpret the solution's meaning.

We view understanding in a way compatible with Piaget (1968). To Piaget, understanding was synonymous with assimilation to a scheme (Thompson 2013). Hence, in our view, test items that prompt students to demonstrate they have assimilated a concept into an appropriate scheme are classified as "understand." Tasks that require understanding could require students to make inferences about the concept by providing examples, making interpretations or comparisons, or providing explanations. For example, the item in Fig. 3 requires students to interpret the meaning of the derivative at a point, the average rate of change, and the definite integral in the context of a situation.

The task in Fig. 3 requires students to demonstrate an understanding by asking them to interpret the meaning of various expressions. Part (a) requires students to interpret the symbols that represent a derivative at a point, part (b) requires students to explain the meaning of average rate of change on a specified interval of a function's domain, and part (c) requires students to interpret integral notation and the meaning of a definite integral. That students must interpret these expressions in the context of a function that defines the relationship between quantities (Thompson 2011) additionally requires understanding. We should caution, however, that tasks might elicit understandings we, or others, do not consider desirable. In general, our coding of items relative to the understandings they elicit was not influenced by our assessment of the utility or desirability of these understandings.

The intellectual behavior of applying understanding involves applying the product of an understanding as opposed to applying a rehearsed procedure. If a student is applying concepts or procedures that are brought to the task, the student must then

Let $r(x)$ represent the total revenue obtained by Company A from selling $x$ items. Interpret the meaning of the following:
a. $r^{\prime}(4,579)$
b. $\frac{r(998)-r(351)}{998-351}$
c. $\int_{2,485}^{10,219} r(x) d x$

Fig. 3 Sample item requiring the cognitive behavior of understanding 
recall those concepts or procedures and apply them. Since we are not able to assess the understandings that students bring to the task, we must ask, "Does the task present the student with an opportunity to demonstrate understandings, and further, does the task necessitate the application of these understandings in order to successfully complete the task?" If so, the item's orientation is "apply understanding."

Tasks that require students to apply understanding often require them to transfer knowledge to a less familiar domain in which surface features of the problem statement cannot serve as cues to trigger the enactment of a rehearsed problem-solving procedure. For example, consider the task in Fig. 4. This task requires students to first identify relationships between relevant varying and constant quantities for the purpose of defining a function to describe how an angle measure and distance are varying together. Students then need to recognize that determining the derivative of the distance with respect to the angle measure will produce a new function that describes the rate of change of the vertical distance that the balloon has traveled with respect to the angle of elevation. Knowing to take the derivative requires that students understand the usefulness of the derivative in determining the speed of the balloon at a moment in time.

Test items that elicit the cognitive behavior of analyzing require students to determine the relationships between constituent components of a mathematical concept, and then establish how these components contribute to the overall structure or purpose of the concept. For instance, consider the task in Fig. 5. The task prompts students to explain the meaning of the limit concept and describe why it is a central idea in the study of calculus; thereby requiring students to determine the relationship between the limit concept and other central concepts such as differentiation and integration. We claim that a task of this type requires a strong understanding of limit in addition to more advanced cognitive structures since students must understand and explain how complex ideas are connected.

\section{Item Representation}

Characterizing the representation of exam items involved classifying both the representation(s) used in the stated task and the representation(s) of the correct solution. Table 2 describes these classifications relative to the task statement and the solution. We also note that both the task statement and the solicited solution can involve multiple representations.

It is important to note that since many tasks can be solved in a variety of ways and with consideration of multiple representations, we coded for the representation requested in the solution by considering only what the task requires for an answer. For

A hot air balloon rising straight up from a level field is tracked by a range finder 500 feet from

the lift-off point. At the moment the range finder's elevation angle is $\pi / 4$, the angle is increasing at the rate of 0.14 radians per minute. How fast is the balloon rising at that moment? (Finney et al., 2007, p. 247).

Fig. 4 Sample item illustrating the contrast between the "recall and apply procedure" and "apply understanding" levels of the item orientation taxonomy 
Write a one-page essay explaining why the concept of limit is a central theme of the course.

Your essay should describe what it means to understand this concept and explain how the concept is related to other ideas in the course.

Fig. 5 Sample item requiring the cognitive behavior of analyzing

example, a problem that asks students to calculate the slope of a tangent line only requires a student to do symbolic work. Accordingly, we would not code "graphical" as a representation of the solution since reasoning graphically is not necessary to solve the problem, even though the problem has graphical meaning.

\section{Item Format}

The third and final strand of the Exam Characterization Framework is item format. The most general distinction of an item's format is whether it is multiple-choice or openended. However, open-ended tasks vary in terms of how they are posed. For instance, the statement of an open-ended task could prompt the student to respond to one

Table 2 Descriptions of item representation categories

\begin{tabular}{lll} 
Item representation Task statement & Solicited solution \\
\hline
\end{tabular}

Applied/modeling The task presents a physical or contextual situation.

Symbolic

Tabular

Graphical

The task conveys information in the form of symbols.

The task provides information in the form of a table.

The task presents a graph.

Definition/theorem The task asks the student to state or interpret a definition or theorem, or presents/cites a definition or theorem.

Proof

The task presents a conjecture or proposition.

Example/ counterexample

The task presents a proposition or statement with the expectation that an example or counterexample is provided.

Explanation what is expected in the students' solution.

The task requires students to define relationships between quantities. The task could also prompt students to define or use a mathematical model to describe information about a physical or contextual situation.

The task requires the manipulation, interpretation, or representation of symbols.

The task requires students to organize data in a table.

The task requires students to generate a graph or illustrate a concept graphically (e.g., draw a tangent line or draw a Riemann sum).

The task requires a statement of a definition or theorem, or an interpretation of a definition or theorem.

The task requires students to demonstrate the truth of a conjecture or proposition by reasoning deductively.

The task requires students to produce an example or counterexample.

The task requires students to explain the meaning of a statement. 
question that has one correct answer. Such an item is similar to a multiple-choice item without the choices and is therefore classified as short answer. In contrast, a broad open-ended task elicits various responses, with each response typically supported by some explanation. The form of the solution in a broad open-ended item is not immediately recognizable when reading the task. In addition to coding tasks as short answer or broad open-ended, we also noted instances in which a task was presented in the form of a word problem. Also, tasks that require students to explain their reasoning or justify their solution can be supplements of short answer or broad open-ended items. We distinguish between explanations and justifications in that explanations are presented in the form of narrative descriptions, using words, and justifications are presented mathematically (e.g., requiring further symbolic or computational work to demonstrate the validity of some previous result). Table 3 contains descriptions of the item format codes.

Table 4 contains the constructs that comprise each of the three dimensions of the Exam Characterization Framework.

The first author trained two graduate students in mathematics to use the Exam Characterization Framework. The graduate students then coded ten randomly selected exams (containing 226 items) so that we could examine inter-rater reliability. Our primary purpose in measuring inter-rater reliability was to ensure that the constructs within the Exam Characterization Framework were defined with sufficient clarity. For this reason, we were satisfied with calculating percent agreement rather than a more formal reliability measure like Krippendorff's alpha or Fleiss' kappa. The first author and the two graduate students achieved a reliability of $89.4 \%$ for the item orientation codes, $73.2 \%$ for the item representation codes, and $92.7 \%$ for the item format codes. We note that the item representation codes were more difficult since coding for item representation involved identifying both the representation of the task statement and the representation of the intended solution, either of which could receive multiple representation codes.

Table 3 Descriptions of item format categories

\begin{tabular}{ll}
\hline Item format & Description \\
\hline Multiple choice & $\begin{array}{l}\text { One question is posed and one answer in a list of choices is correct. The student is } \\
\text { prompted to select the correct answer among the choices. } \\
\text { The item asks the student to respond to one question that has one correct answer. The } \\
\text { student can anticipate the form of the solution merely by examining the task-this is } \\
\text { similar to a multiple-choice item without the choices. }\end{array}$ \\
Broad open-ended & $\begin{array}{l}\text { There are multiple ways of expressing the answer. The form of the solution is also not } \\
\text { immediately recognizable upon immediate inspection of the task. }\end{array}$ \\
A & $\begin{array}{l}\text { A word problem is posed in a contextual setting, and prompts students to create an } \\
\text { algebraic, tabular and/or graphical model to relate specified quantities in the } \\
\text { problem, and could also prompt students to make inferences about the quantities } \\
\text { in the context using the model. Note that a word problem can be posed as either } \\
\text { short answer or broad open-ended or multiple choice. Hence, we code a task as a } \\
\text { word problem in addition to identifying it as either short answer or broad open-ended. }\end{array}$
\end{tabular}

Explain $(E)$ and justify $(J)$ are subcodes of Item Format 
Table 4 Summary of exam characterization framework dimensions

\begin{tabular}{|c|c|c|}
\hline Exam characterization dimension & Categories & \\
\hline Item orientation & $\begin{array}{l}\text { Remember } \\
\text { Recall and apply procedure } \\
\text { Understand } \\
\text { Apply understanding } \\
\text { Analyze } \\
\text { Evaluate } \\
\text { Create }\end{array}$ & \\
\hline Item representation & $\begin{array}{l}\text { Applied/modeling } \\
\text { Symbolic } \\
\text { Tabular } \\
\text { Graphical } \\
\text { Definition/theorem } \\
\text { Proof } \\
\text { Example/counterexample } \\
\text { Explanation }\end{array}$ & \\
\hline \multirow[t]{2}{*}{ Item format } & Multiple choice & \\
\hline & Open-ended & $\begin{array}{l}\text { Short answer } \\
\text { Word problem } \\
\text { Broad open-ended }\end{array}$ \\
\hline
\end{tabular}

Even though we have evidence that our coding framework is highly reliable if used by knowledgeable coders, we do not advocate that others attempt to use this framework to code Calculus I exams, or exams in another mathematics content area in mathematics (e.g., precalculus), without establishing consistency among coders. We believe the process of familiarizing oneself with the items in one's data set and the constructs that comprise our coding framework is necessary, and that in any setting a high level of consistency among coders should be established prior to generating coded results for use in analyses. In our case, we had established consistency between the first two authors of this article for the purpose of refining the constructs and providing clearly stated descriptions of our meaning of each construct; however, we assured consistency in coding since the first author coded all items on all 150 exams in our sample. It was also important that the first author had a clear understanding of the intended meaning of all constructs in our framework and repeatedly coded items that were particularly challenging to code.

\section{Limitations}

There are several limitations of our framework, as well as our use of it to characterize post-secondary Calculus I final exams. The first is shared by some of the existing frameworks in the literature. Identifying the cognitive demand that exam items elicit in students is problematic since we have no knowledge of the instruction the students taking any particular exam experienced. As a result, a seemingly rote task has the potential to be highly novel for students who have not been exposed to the procedure or method for solving the task. Conversely, tasks that might appear to require an understanding of a particular concept are amenable to being proceduralized if the solution 
method is practiced repetitiously. We spoke earlier of our intention to code for the minimal reasoning practices and solution representations that a task requires. To do this with a reasonable degree of validity required us to assess minimal reasoning practices and solution representations relative to our model of the epistemic collegiate calculus student. ${ }^{3}$ Additionally, our coding was informed by our knowledge of those concepts in the calculus curriculum that are generally proceduralized (e.g., differentiation, integration, limit evaluation). The first two authors have taught the entire calculus sequence multiple times and have researched student learning in Calculus I. These common experiences resulted in the authors reaching a common understanding of what constituted viable models of the epistemic calculus student and the general calculus curriculum.

A second limitation is that we do not have data indicating how the exams were graded. Accordingly, we present our results as percentages of exam items classified within each of the respective categories of the Exam Characterization Framework. This method of computing results does not account for the reality that particular exam items might represent a higher portion of the exam grade, and thus be valued by the instructor to a greater extent.

A third limitation is that we coded each part of an item as an individual item. For instance, we coded a task that has parts (a), (b), and (c) as three distinct items. This is a limitation in the sense that some instructors have a tendency to subdivide tasks while others do not, and our Exam Characterization Framework does not identify the number of mathematical procedures or understandings needed to complete a task. However, our large sample size somewhat mediates this concern. While our decision to code each part of an exam question as an individual item has its disadvantages, a reviewer of a previous version of this manuscript suggested that this decision might have contributed to our achieving a more accurate characterization of the final exams since an instructor's subdivision of tasks might constitute a form of scaffolding intended to provide students with explicit intermediate goals.

In the introduction of this paper, we reveal our assumption that the content of a final exam reflects an instructor's expectations for students' learning. We were less explicit, however, about our assumption that a final exam is a viable representation of an instructor's assessment practices. While both assumptions were crucial to the design of this study, we would like to preface our results by acknowledging three limitations of these assumptions. First, many instructors administer departmental exams, which are typically developed by course coordinators who solicit the input of the faculty to various extents. A final exam may therefore not be under the direct control of individual instructors. Second, mathematics departments often experience pressure from client departments and higher administration to lower failure rates in introductory courses. This pressure might compel instructors and course coordinators to create exams that are not as cognitively demanding than they would otherwise be. Third, instructors might use more conceptually oriented tasks to support students' learning of content that are not reflected in exams that are designed to assess learning

\footnotetext{
3 "Epistemic collegiate calculus student" is used in the Piagetian sense and is characterized by our idealized abstraction of the college calculus student.
} 
outcomes. All of these factors likely contribute to skewing the makeup of final exams toward more procedural tasks. We encourage the reader to interpret our results in light of these limitations.

\section{Results}

Data analysis in this study consisted of three phases. In the first phase, we developed the Exam Characterization Framework discussed above and used it to code the 150 randomly selected exams in our sample. In the second phase, we compared the coded exam data with data obtained from a post-term instructor survey with the intention of determining the extent to which our characterization of the exams corresponds with instructors' perceptions of their exams relative to their conceptual orientation. In the third phase, we coded 13 Calculus I final exams administered in U.S. colleges and universities in 1986/87 and compared these results to those of our random sample of 150 exams.

\section{Characteristics of Post-Secondary Calculus I Exams}

The results from coding the 150 Calculus I final exams using the item orientation taxonomy revealed that only $14.72 \%$ of the 3735 exam items required students to demonstrate an understanding of an idea or procedure. More specifically, $6.51 \%$ of the items required students to employ the cognitive behavior of remembering, $78.70 \%$ of the items required students to recall and apply a rehearsed procedure, $4.42 \%$ required students to demonstrate an understanding, and $10.30 \%$ of the exam items required students to apply their understanding while solving a problem. These results indicate that the vast majority of exam items $(85.21 \%)$ could be solved by simply retrieving rote knowledge from memory, or recalling and applying a procedure, requiring no understanding of an idea or why a procedure is valid.

Of the 150 exams coded, $90 \%$ of them had at least $70 \%$ of their items coded at the "remember" or "recall and apply procedure" levels of the item orientation taxonomy. Additionally, only $2.67 \%$ of exams had $40 \%$ or more of the items requiring students to demonstrate or apply understanding. Table 5 contains the coding results for the item orientation taxonomy.

The box plots in Fig. 6 provide a summary of the percentage of exam items per exam within each category of the item orientation taxonomy. Consistent with the aggregate

Table 5 Percentage of items within each category of the item orientation taxonomy

\begin{tabular}{ll}
\hline Item orientation & $\%$ \\
\hline Remember & 6.51 \\
Recall and apply procedure & 78.70 \\
Understand & 4.42 \\
Apply understanding & 10.30 \\
Analyze & 0.11 \\
Evaluate & 0 \\
Create & 0 \\
\hline
\end{tabular}




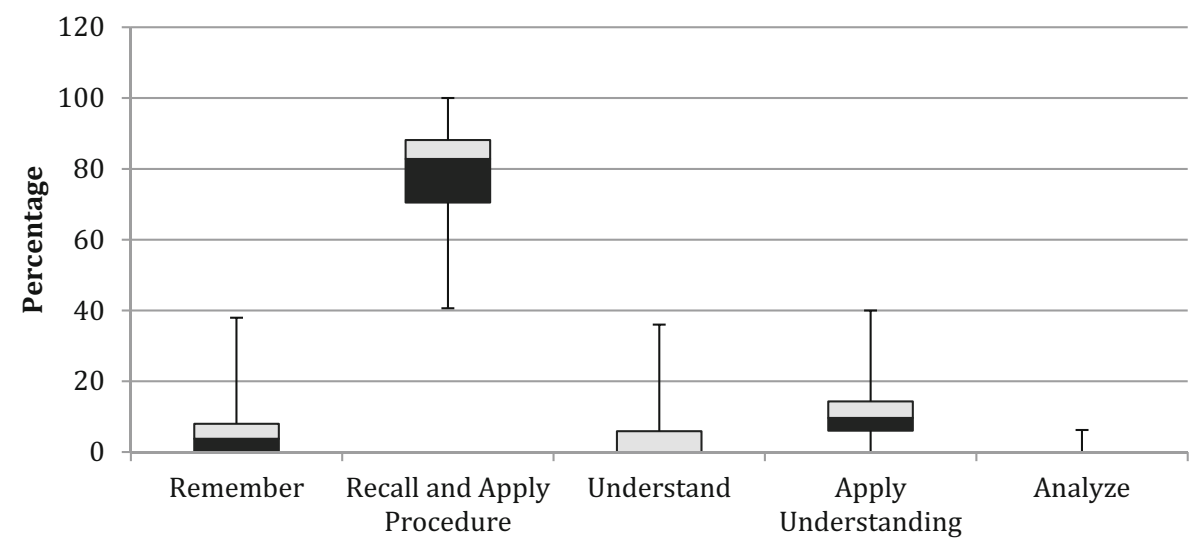

Item Orientation Codes

Fig. 6 Box plots representing the percentage of items per exam within each category of the item orientation taxonomy

data in Table 5, we observe that the highest percentage of exam items per exam required the cognitive behavior of recalling and applying a procedure, with consistent distributions for the "remember," "understand," and "apply understanding" categories of the taxonomy. We also observe that the minimum value for the distribution of the percentage of exam items requiring students to recall and apply a procedure exceeds the maxima of all other distributions in the plot-suggesting that any single exam contained more items requiring students to recall and apply a procedure than demonstrate any other cognitive behavior.

We calculated the percentage of items within each category of the item orientation taxonomy for each post-secondary institution type, and the results are provided in Table 6 . The highest percentage of items requiring the cognitive behavior of recalling and applying procedures were those from community colleges $(84.4 \%)$ while the lowest were from national liberal arts colleges $(69.6 \%$ )- those that emphasize undergraduate education and award at least half of their degrees in the liberal arts fields of study. It is also noteworthy that national universities contained the highest percentage of items

Table 6 Percentage of items within each item orientation by post-secondary institution classification

\begin{tabular}{llllll}
\hline Item orientation & $\begin{array}{l}\text { National } \\
\text { university }\end{array}$ & $\begin{array}{l}\text { Regional } \\
\text { university }\end{array}$ & $\begin{array}{l}\text { Community } \\
\text { college }\end{array}$ & $\begin{array}{l}\text { National liberal } \\
\text { arts college }\end{array}$ & $\begin{array}{l}\text { Regional } \\
\text { college }\end{array}$ \\
\hline Remember & $5.53 \%$ & $9.33 \%$ & $4.25 \%$ & $8.77 \%$ & $13.46 \%$ \\
Recall and apply procedure & $78.9 \%$ & $77.6 \%$ & $84.4 \%$ & $69.6 \%$ & $78.8 \%$ \\
Understand & $4.6 \%$ & $3.0 \%$ & $3.7 \%$ & $10.5 \%$ & $3.8 \%$ \\
Apply understanding & $10.8 \%$ & $10.0 \%$ & $7.6 \%$ & $10.5 \%$ & $3.8 \%$ \\
Analyze & $0.1 \%$ & $0.1 \%$ & $0.0 \%$ & $0.0 \%$ & $0.0 \%$ \\
Evaluate & $0.0 \%$ & $0.0 \%$ & $0.0 \%$ & $0.0 \%$ & $0.0 \%$ \\
Create & $0.0 \%$ & $0.0 \%$ & $0.0 \%$ & $0.0 \%$ & $0.0 \%$ \\
\hline
\end{tabular}


requiring students to apply their understanding (10.8\%) while the lowest were from regional colleges $(3.8 \%$ ) - those that focus on undergraduate education, awarding less than half of their degrees in liberal arts fields of study. Moreover, national liberal arts colleges contained the highest percentage of items at the "understand" level of the item orientation taxonomy or higher $(21 \%)$, while regional colleges contained the highest percentage of items at the "remember" or "recall and apply procedure" levels (96.26\%).

In terms of item representation, exam items were predominantly stated symbolically $(73.70 \%)$ or required a symbolic solution $(89.4 \%)$. Few items prompted students for information in the form of a table $(1.02 \%)$, presented a proposition or statement with the expectation that students provide an example or counterexample $(0.59 \%)$, or presented a conjecture or proposition with the expectation that students construct a proof $(1.29 \%)$. Table 7 contains percentages of exam items within each of the item representation categories.

The majority of Calculus I final exam items in our sample were stated symbolically and required a symbolic solution (65.45\%). Moreover, only $6.94 \%$ of the items presented a physical or contextual situation and solicited a solution in which students were required to define relationships between quantities or use a mathematical model to describe information about a physical or contextual situation. Taking into consideration the increased emphasis in calculus on making connections among multiple representations (i.e., words, symbols, tables, and graphs), it is noteworthy that only $9.59 \%$ of exam items were stated exclusively as "applied/ modeling," "symbolic," "tabular," or "graphical" while requiring the solution to be presented in a different representation. Table 8 outlines the most prevalent combinations of item representations in our sample. ${ }^{4}$

Our analysis also indicated that introductory calculus final exams seldom include tasks that are stated in the context of a real-world situation. Our coding revealed that $38.67 \%$ of the coded exams had less than $5 \%$ of the items classified as word problems, in either the "short answer" or "broad openended" format categories. Further, only $22 \%$ of the coded exams had more than $10 \%$ of the exam's test items classified as a word problem in the "short answer" or "broad open-ended" format categories. It is also noteworthy that $18 \%$ of the exams contained no word problems. We provide the coding results from the item format dimension of the ECF in Table 9.

In the final analysis, results from coding the 150 randomly selected exams with the Exam Characterization Framework revealed that the Calculus I final exams in our random sample require low levels of cognitive demand, contain few problems stated in a real-world context, rarely elicit explanation, and seldom require students to demonstrate or apply their understanding of the course's central ideas.

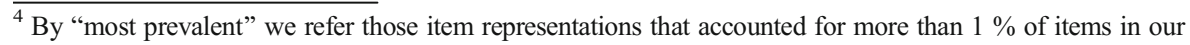
sample.
} 
Table 7 Percentage of items within item representation categories

\begin{tabular}{lrlr}
\hline Item representation (task) & \multicolumn{1}{l}{$\%$} & Item representation (solution) & \multicolumn{1}{c}{$\%$} \\
\hline Applied/modeling & 13.20 & Applied/modeling & 6.96 \\
Symbolic & 73.70 & Symbolic & 89.40 \\
Tabular & 1.02 & Tabular & 0.19 \\
Graphical & 10.40 & Graphical & 5.70 \\
Definition/theorem & 3.51 & Definition/theorem & 4.36 \\
Proof & 1.29 & Proof & 1.53 \\
Example/counterexample & 0.59 & Example/counterexample & 0.59 \\
Explanation & 2.36 & Explanation & 2.36 \\
\hline
\end{tabular}

\section{Contrast Between Instructors' Beliefs and Coding Results}

To address the second focus of our study, we coordinated the results of the exam codes and instructors' responses to a post-term instructor survey. We devoted particular attention to identifying inconsistencies between our findings about the characteristics of the Calculus I final exams and instructors' perceptions of their exams.

Figure 7 provides the distribution of instructors' survey responses to the question, "How frequently did you require students to explain their thinking on exams?" Responses ranged from 1 (not at all) to 6 (very often). We note that we would very likely have coded an exam item requiring explanation as either "understand" or "apply understanding" using the item orientation taxonomy. Results from the item format codes indicate that a total of $3.05 \%$ of all items coded $(N=3735)$ required an explanation. Further, only $14.72 \%$ of all exam items were coded as "understand" or "apply understanding" using the item orientation taxonomy. However, $68.18 \%$ of all instructors who submitted exams that were part of our sample selected either 4, 5, or 6 on this survey item, indicating that these instructors claim to frequently require their students to explain their thinking on exams. These data reveal that the instructors' Calculus I final exams do not align with their perceptions of them relative to the extent to which students are required to explain their thinking.

Similarly, there were also discrepancies between our characterization of the final exams and survey responses with respect to the percentage of exam items that emphasized skills and methods for executing computations. Figure 8 displays the distribution of instructors' responses to the survey question, "On a typical exam, what percentage of the points focused on skills and methods for carrying out computations?" The median response was $50 \%$. Our coding results, however, indicate that $78.7 \%$ of exam items require students to recall and apply a procedure. Additionally, $89.4 \%$ of all exam items required students to perform symbolic computations.

In summary, these data reveal that there is a misalignment between our characterization of Calculus I final exams and instructors' perceptions of their exams relative to the extent to which students are asked to explain their thinking and the percentage of exam items that focus on skills and methods for carrying out computations. 
Table 8 Percentage of exam items within item representation categories

\begin{tabular}{llr}
\hline Item representation (task) & Item representation (solution) & $\%$ \\
\hline Symbolic & Symbolic & 65.45 \\
Applied/modeling & Applied/modeling; Symbolic & 6.86 \\
Graphical & Symbolic & 6.72 \\
Applied/modeling & Symbolic & 4.90 \\
Symbolic & Graphical & 2.01 \\
Definition & Definition & 1.90 \\
Symbolic & Symbolic; Graphical & 1.61 \\
\hline
\end{tabular}

\section{Correlating Item Orientation with Representation and Format}

To determine if particular item representations or formats necessitated higher-order cognitive activity, we calculated the percentage of item representations and item format types within each item orientation category. Table 10 documents the percentage of the most common item representation types (used in the task and asked for in the solution) for the first four levels of the item orientation taxonomy. ${ }^{5}$

As Table 10 indicates, the highest percentage of items $(30.20 \%)$ that required the cognitive behavior of remembering were stated symbolically and required a symbolic solution. Similarly, the vast majority of tasks that required students to recall and apply a procedure $(78.98 \%)$ were stated symbolically and required a students to produce a solution in the form of mathematical symbols. We also observed that the percentage of items on an exam that were stated symbolically and solicited a symbolic solution decreased as tasks demanded higher levels of cognitive behavior $(10.30 \%$ in the "understand" category, $5.50 \%$ in the "apply understanding" category, and $0 \%$ in the "analyze" category").

It is also noteworthy that items presented in a contextual or physical situation (i.e., applied/modeling) were most prevalent among items that required students to apply understanding (70.94\%). The applied/modeling tasks also represented a significant percentage of items that required students to demonstrate understanding $(10.30 \%)$. Moreover, $20 \%$ of items that required students to demonstrate understanding presented information in the form of a graph and solicited symbolic work in the solutionalthough these items often required explanation or justification. Thus, in the majority of items that required students to demonstrate understanding or apply understanding, students were required to interpret information from a graphical representation or applied setting. As an example, consider the task in Fig. 9 stated graphically.

All parts of the task in Fig. 9 require students to demonstrate understanding since they are asked to infer the behavior of a function $f$ and a function $\int f(x) d x$ by examining

\footnotetext{
$\overline{5}$ By "most common," we refer to an exclusion of item representations that represented less than $5 \%$ of the items within a specific item orientation category.

6 The percentages of item representations within the "analyze" category of the item orientation taxonomy are not provided in Table 11 as a result of the small number of items within this category $(n=4)$.
} 
Table 9 Percentage of items within each item format category

\begin{tabular}{ll}
\hline Item format & $\%$ \\
\hline Multiple choice & 11.70 \\
Multiple choice (explain) & 0.59 \\
Multiple choice (justify) & 0.19 \\
Multiple choice (word problem) & 0.40 \\
Short answer & 76.10 \\
Short answer (explain) & 2.38 \\
Short answer (justify) & 1.04 \\
Short answer (word problem) & 6.05 \\
Broad open-ended & 1.23 \\
Broad open-ended (explain) & 0.08 \\
Broad open-ended (justify) & 0 \\
Broad open-ended (word problem) & 0.03 \\
\hline
\end{tabular}

only the graphical behavior of the function $f^{\prime}$; thereby requiring students to attend to the meaning of the derivative function and what the indefinite integral represents.

Table 11 indicates the percentage of various item formats within the first four categories of the item orientation taxonomy. ${ }^{7}$ We find it notable that short answer items requiring students to provide an explanation accounted for a much higher percentage of items eliciting the cognitive behavior of understanding $(15.15 \%)$ than items requiring the recollection and application of a procedure $(1.16 \%)$ or items simply prompting students to remember $(0.41 \%)$. Moreover, $97.93 \%$ of word problems - whether stated in the form of multiple choice, short answer, or broad open-ended - provided students with the opportunity to apply their understanding. Finally, we observe that $6.50 \%$ of the total number of items prompting students to provide an explanation or justification required only the cognitive behavior of remembering, whereas $27.64 \%$ required students to recall and apply a procedure, $31.71 \%$ required students to demonstrate understanding, and $34.15 \%$ provided students with the opportunity to apply their understanding.

These data suggest that items presented in the form of word problem or prompting students to provide an explanation or justification for their work elicit higher-order cognitive behavior.

\section{Comparing the Cognitive Demand of Our Sample with Final Exams from 1986/87}

The results presented thus far reveal that our sample of post-secondary Calculus I final exams require low levels of cognitive engagement, rarely make use of real-world contexts, seldom elicit explanations or justifications, and do not provide students with opportunities to demonstrate or apply their understanding. These results are particularly surprising considering the considerable amount of attention devoted to the conceptual teaching of calculus throughout the past 25 years. This led us to consider how

\footnotetext{
${ }^{7}$ We include only those item formats representing more than $1 \%$ of the items within the first four item orientation categories.
} 


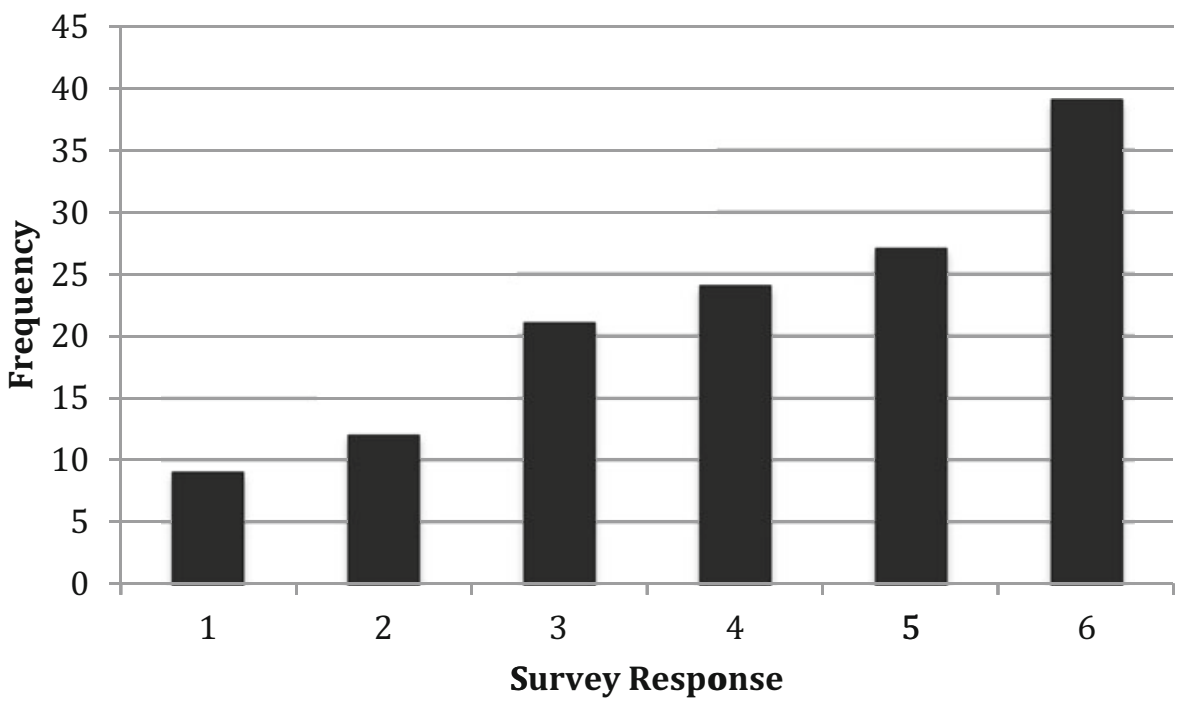

Fig. 7 Instructors' response to the question, "How frequently did you require students to explain their thinking on exams?"

contemporary Calculus I final exams compare with those administered prior to the calculus reform movement. We were able to achieve this comparison by coding Calculus I final exams administered in 1986/87 to students enrolled in first semester

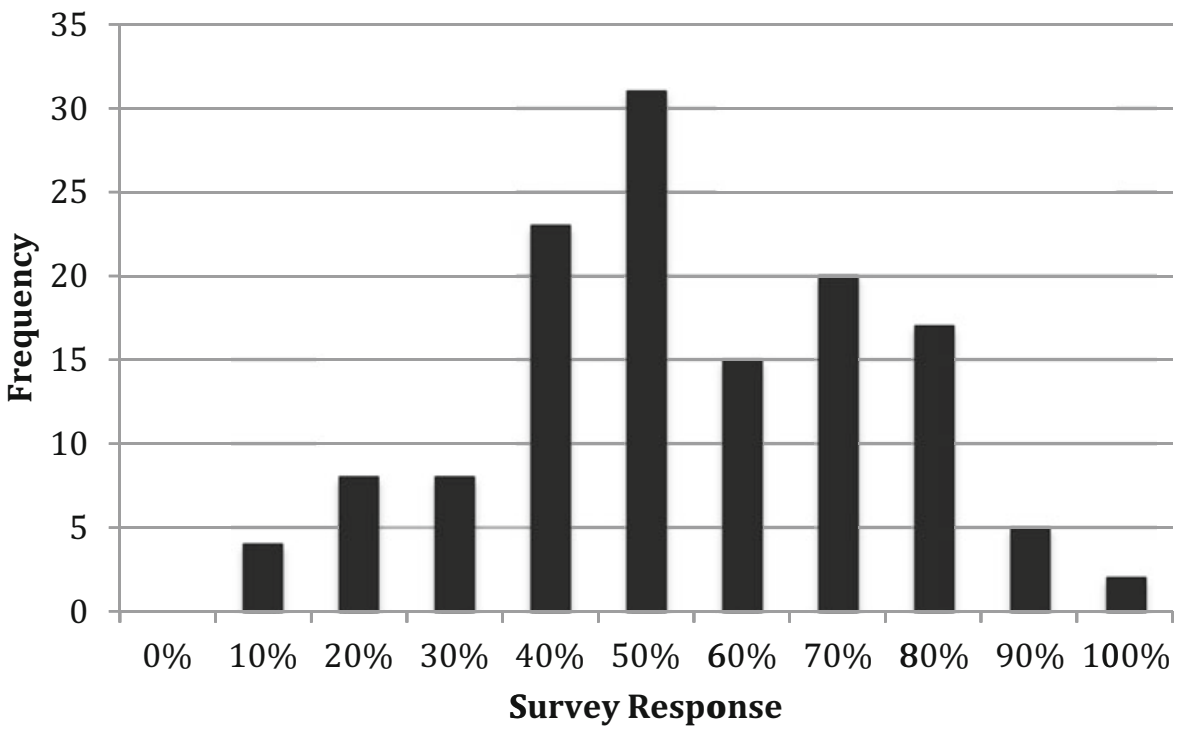

Fig. 8 Instructors' response to the question, "On a typical exam, what percentage of the points focused on skills and methods for carrying our computations?" 
Table 10 Percentage of item representations within each category of the item orientation taxonomy

\begin{tabular}{lllr}
\hline Item orientation & Item representation (task) & Item representation (solution) & \multicolumn{1}{c}{$\%$} \\
\hline Remember & Symbolic & Symbolic & 30.20 \\
& Definition & Definition & 27.76 \\
& Graphical & Symbolic & 21.63 \\
Recall and apply procedure & Symbolic & Symbolic & 78.98 \\
& Applied/modeling & Symbolic & 5.20 \\
& Graphical & Symbolic & 5.10 \\
Understand & Graphical & Symbolic & 20.00 \\
& Symbolic & Explanation & 13.33 \\
& Symbolic & Symbolic & 10.30 \\
& Definition & Explanation & 7.27 \\
& Applied/modeling & Explanation & 10.30 \\
Apply understanding & Applied/modeling & Applied/modeling; Symbolic & 65.18 \\
& Applied/modeling & Symbolic & 5.76 \\
& Symbolic & Symbolic & 5.50 \\
& Symbolic & Definition & 5.45 \\
\hline
\end{tabular}

calculus from a variety of post-secondary institutions across the United States, from large doctoral granting universities to small 2-year colleges (Steen 1988). The 13 Calculus I final exams (with 354 total items) we coded were included in the appendix of Steen (1988). We provide the results from coding this sample of 1986/87 exams relative to item orientation in Table 12.

Our analysis revealed that there is a statistically significant difference between the proportion of items requiring students to recall and apply a procedure in our sample of 150 calculus exams and the 13 Calculus I final exams from 1986/87. ${ }^{8}$ The difference between these two data sets in the proportion of items within the "apply understanding" category of the item orientation taxonomy, however, is not statistically significant. While there is significantly less emphasis on recalling and applying procedures in contemporary Calculus I exams, compared to those administered 25 years ago, our results suggest that the proportion of exam items eliciting the cognitive behavior of applying understanding has not significantly changed. This finding is surprising considering the tremendous effort devoted to the conceptual teaching of calculus since the initiation of the calculus reform movement in the late 1980s.

Calculus reform initiatives have emphasized the importance of developing students' reasoning with multiple representations. One would expect, then, that more recent Calculus I exam items would require students to interpret information presented in a particular representation (e.g., applied/modeling) and translate it to another (e.g., graphical). Table 13 provides the percent of exam items from the Calculus I final exams from 1986/87 within various item representation categories.

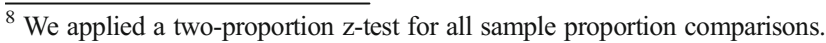


The following graph represents the graph of the derivative $f^{\prime}$ of a function $f$ that is defined on the interval $[-5,6]$. Using this graph, answer the following questions about the function $f$ and the function $\int f(x) d x$.

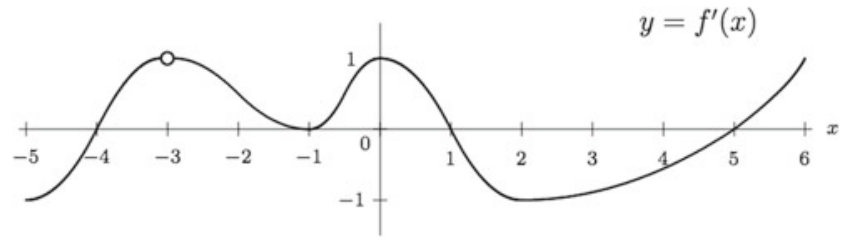

Note: the empty circle represents a hole in the graph.

(f) On what intervals is $f$ decreasing?

(g) On what intervals is $\int f(x) d x$ increasing?

(h) On what intervals is $f$ concave up?

(i) What are the critical points of $\int f(x) d x$ ?

(j) For the critical points identified in part (d), determine whether it is a local maximum, local minimum, or neither and explain your response.

Fig. 9 Item stated graphically that elicits the cognitive behavior or understanding

Table 13 contrasts the percent of exam items from the 1986/87 sample within each item representation category with those from our sample of 150 exams from 2010/11. These data reveal that a higher percentage of items on the 1986/87 final exams were stated symbolically and required students to interpret, represent, or manipulate symbols in the solution than items in our initial sample. However, a higher percentage of items on the exams from 1986/87 presented a physical or contextual situation and required students to define the relationship between quantities. We illustrate a comparison of item representations among exams from 1986/87 and our original sample in Fig. 10.

\section{Discussion}

In an effort to address high failure rates in college calculus, the calculus reform movement in the United States initiated a number of instructional and curricular innovations that sought to deepen students' conceptual understanding of the course's foundational ideas (Ganter 2001). With this increase in conceptual focus, one might expect that calculus exams would include more items that assess students' understanding and ability to apply the key concepts of calculus. Our analysis suggests this is not the case. 
Table 11 Percentage of item formats within each category of the item orientation taxonomy

\begin{tabular}{llr}
\hline Item orientation & Item format & \multicolumn{1}{c}{$\%$} \\
\hline Remember & Short answer & 62.04 \\
& Multiple choice & 32.65 \\
& Multiple choice (explain) & 3.27 \\
& Short answer & 86.87 \\
& Multiple choice & 10.58 \\
& Short answer (explain) & 1.16 \\
Understand & Short answer & 36.97 \\
& Multiple choice & 24.85 \\
& Short answer (explain) & 15.15 \\
& Broad open-ended & 13.94 \\
& Multiple choice (explain) & 5.45 \\
& Multiple choice (justify) & 1.82 \\
& Broad open-ended (explain) & 1.21 \\
Apply understanding & Short answer (word problem) & 56.28 \\
& Short answer & 22.25 \\
& Short answer (explain) & 7.59 \\
& Multiple choice (word problem) & 3.93 \\
& Broad open-ended & 3.66 \\
& Multiple choice & 2.09 \\
& Short answer (justify) & 1.83 \\
& Short answer (word problem/justify) & 1.57 \\
\hline
\end{tabular}

Our examination of 150 modern Calculus I final exams revealed that these exams primarily assess students' ability to recall and apply procedures; there is little focus on assessing students' understanding, with $85.21 \%$ of the 3735 exam items being solvable by simply retrieving rote knowledge from memory, or recalling and applying a procedure. The Calculus I final exams in our sample rarely make use of real-world contexts, seldom elicit explanation or justification, and provide few opportunities for

Table 12 Percentage of 1986/87 exam items within each item orientation category

\begin{tabular}{lll}
\hline Item orientation & $1986 / 87(\%)$ & $2010 / 11(\%)$ \\
\hline Remember & 3.67 & 6.51 \\
Recall and apply procedure & 87.01 & 78.70 \\
Understand & 1.13 & 4.42 \\
Apply understanding & 8.19 & 10.30 \\
Analyze & 0 & 0.11 \\
Evaluate & 0 & 0 \\
Create & 0 & 0 \\
\hline
\end{tabular}


Table 13 Percentage of 1986/87 exam items within each item representation category

\begin{tabular}{llll}
\hline Item representation (task) & Item representation (solution) & $1986 / 87$ (\%) & 2010/11 (\%) \\
\hline Symbolic & Symbolic & 74.01 & 65.45 \\
Applied/modeling & Applied/modeling; Symbolic & 12.71 & 6.86 \\
Symbolic & Symbolic; Graphical & 4.80 & 1.61 \\
Graphical & Symbolic & 3.11 & 6.72 \\
Definition & Definition & 1.69 & 1.90 \\
Symbolic & Graphical & 1.13 & 2.01 \\
Symbolic; Definition & Symbolic & 0.56 & 0.11 \\
Tabular & Symbolic & 0.56 & 0.51 \\
Symbolic & Applied/modeling; Symbolic & 0.28 & 0 \\
Symbolic; Tabular; Graphical & Symbolic & 0.28 & 0 \\
Graphical & Graphical & 0.28 & 0.88 \\
Symbolic & Symbolic; Tabular; Graphical & 0.28 & 0 \\
Symbolic & Symbolic; Definition & 0.28 & 0 \\
\hline
\end{tabular}

students to demonstrate or apply their understanding. Of the 150 exams we coded, $90 \%$ had $70 \%$ or more of the exams' items coded as "remember" or "recall and apply procedure." In contrast, only $2.67 \%$ of the 150 exams had $40 \%$ or more of the items requiring students to demonstrate or apply understanding.

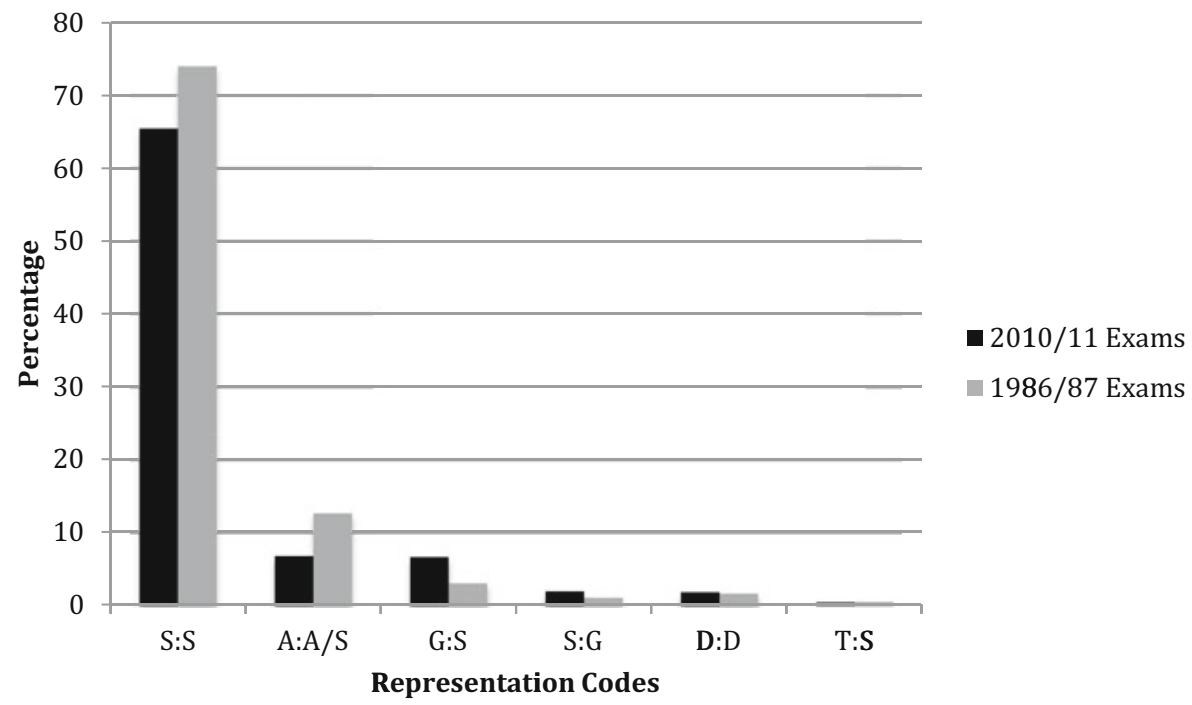

Fig. 10 Comparison of item representations among exams from 1986/87 and our original sample. S:S represents symbolic task representation and symbolic solution representation; A:A/S represents applied/ modeling task representation and applied/modeling and symbolic solution representation; G:S represents graphical task representation and symbolic solution representation; S:G represents symbolic task representation and graphical solution representation; D:D represents definition task representation and definition solution representation; T:S represents tabular task representation and symbolic solution representation 
As a result of the low percentage of exam items at the "understand" or "apply understanding" levels of the item orientation taxonomy, we conclude that a large percentage of exam items failed to provide insight into how students understand the concepts on which their computational or procedural work is based. Hence, these results suggest that a large majority of Calculus I final exams being administered in colleges and universities in the United States promote memorization of procedures for answering specific problem types and do not encourage students to understand or apply concepts of elementary calculus.

Our analysis further demonstrates that Calculus I final exams in U.S. colleges and universities have changed very little in the past 25 years relative to the percentage of exam items that require students to apply their understanding of foundational concepts. Since final course exams commonly reflect the level of mastery and understanding students have attained at the end of the course, these data suggest that the calculus reform movement of the late 1980s has had little effect on what is being assessed in current Calculus I courses in U.S. postsecondary institutions.

We also found that there exist inconsistencies between our characterization of postsecondary Calculus I final exams and instructors' perception of the nature of the exams they administer to their students. Instructors' report that their exams require students to demonstrate and apply understandings. Our analyses revealed that a very high percentage of exam items focus on skills and methods for carrying out computations, while a very low percentage of items prompt students to explain their thinking.

If exams are predominantly based on measures of low level cognitive behaviors such as "remember," or "recall and apply procedure," students are likely to develop perceptions about mathematics as not being about understanding and applying ideas to solve novel problems. This focus on procedures has been reported to be uninteresting to some more capable students who enjoy understanding and reasoning through nonroutine problems (Thompson et al. 2007).

We acknowledge that our discussion of this study's main findings reveal the high value we place on exam items that assess students' understanding of foundational calculus concepts, as well as their ability to apply these understandings to solve novel problems. While we recognize the essential role of procedural fluency in calculus assessment, our privileging of conceptual exam items derives from our own research and from our review of the literature on students' learning of ideas in introductory calculus (e.g., Carlson and Rasmussen 2008). This research has revealed that Calculus I students are generally not developing conceptual understanding of the course's central ideas, which affects the likelihood that they will succeed in calculus and courses beyond calculus. Procedural fluency has its place in calculus assessment but as we have shown, the relative frequencies of procedural and conceptual exam items has not changed much in 25 years of calculus reform efforts. We believe that assessment practices in introductory calculus should reflect the objectives for students' learning advocated in the mathematics education literature as well as those promoted by the calculus reform movement.

We encourage Calculus I instructors and mathematics departments to contemplate the role of their Calculus I final exams in developing students' mathematical abilities, and to consider whether their exams are supporting the development of students' mathematical understandings and thinking. In many cases this might require departments to examine their Calculus I curriculum and instruction. Some might also find it 
useful to consult the mathematics education literature (e.g., Carlson and Rasmussen 2008) that discusses what is involved in understanding foundational calculus ideas, and what studies have revealed about the process of understanding and learning to use these ideas to solve novel problems. We also encourage the use of our Exam Characterization Framework in pre-service teaching programs and graduate teaching assistant workshops as a didactic tool to assist teachers in constructing assessments that afford students the opportunity to demonstrate their understanding.

Acknowledgments This research was supported by National Science Foundation Grant DRL-0910240. Any recommendations or conclusions stated here are those of the authors and do not necessarily reflect official positions of the NSF. We thank SIGMAA on RUME for the opportunity to present a previous version of this manuscript at their 15th annual conference. We also thank Chester Ismay for assisting us in using quantitative data analysis software.

\section{References}

Anderson, L. W., \& Krathwohl, D. R. (Eds.). (2001). A taxonomy for learning, teaching, and assessing: A revision of Bloom's taxonomy of educational objectives. New York: Longman.

Bergqvist, E. (2007). Types of reasoning required in university exams in mathematics. Journal of Mathematical Behavior, 26, 348-370.

Bloom, B. S., et al. (Eds.). (1956). Taxonomy of educational objectives: Part 1, cognitive domain. New York: Longman Green.

Boesen, J., Lithner, J., \& Palm, T. (2006). The relation between test task requirements and the reasoning used by students. Umeå: Department of Mathematics, Umeå University.

Carlson, M. P., \& Rasmussen, C. (Eds.). (2008). Making the connection: Research and teaching in undergraduate mathematics education. Washington: Mathematical Association of America.

Charalambous, C., Delaney, S., Hsu, A., \& Mesa, V. (2010). The addition and subtraction of fractions in the textbooks of three countries: a comparative analysis. Mathematical Thinking and Learning, 12(2), 117151.

Finney, R. L., Demana, F. D., Waits, B. K., \& Kennedy, D. (2007). Calculus: Graphical, numerical, algebraic (3rd ed.). Boston: Pearson Prentice Hall.

Ganter, S. L. (Ed.). (2001). Changing calculus:A report on evaluation efforts and national impact from 19881998. Washington DC: Mathematical Association of America.

Gierl, M. J. (1997). Comparing cognitive representations of test developers and students on a mathematics test with Bloom's taxonomy. The Journal of Educational Research, 91(1), 26-32.

Krathwohl, D. R. (2002). A revision of Bloom's taxonomy: an overview. Theory Into Practice, 41(4), 212218.

Li, Y. (2000). A comparison of problems that follow selected content presentations in American and Chinese mathematics textbooks. Journal for Research in Mathematics Education, 31(2), 234-241.

Lithner, J. (2000). Mathematical reasoning in task solving. Educational Studies in Mathematics, 41(2), 165190.

Lithner, J. (2003). Students' mathematical reasoning in university textbook exercises. Educational Studies in Mathematics, 52(1), 29-55.

Lithner, J. (2004). Mathematical reasoning in calculus textbook exercises. The Journal of Mathematical Behavior, 23, 405-427.

Mesa, V. (2010). Strategies for controlling the work in mathematics textbooks for introductory calculus. In F. Hitt, D. Holton \& P. W. Thompson (Eds.), CBMS Issues in Mathematics Education (Vol. 16, pp. 235261). Providence, RI: AMS.

Mesa, V., Suh, H., Blake, T., \& Whittemore, T. (2012). Examples in college algebra textbooks: opportunities for students' learning. Problems, Resources, and Issues in Mathematics Undergraduate Studies, 23(1), 76-105.

Palm, T., Boesen, J., \& Lithner, J. (2006). The requirements of mathematical reasoning in upper secondary level assessments. Umeå: Departmetn of Mathematics, Umeå University. 
Piaget, J. (1968). Six psychological studies. New York: Vintage Books.

Senk, S. L., Beckman, C. E., \& Thompson, D. R. (1997). Assessment and grading in high school mathematics classrooms. Journal for Research in Mathematics Education, 28(2), 187-215.

Smith, G., Wood, L., Coupland, M., Stephenson, B., Crawford, K., \& Ball, G. (1996). Constructing mathematical examinations to assess a range of knowledge and skills. International Journal of Mathematics Education in Science and Technology, 27(1), 65-77.

Steen, L. (Ed.). (1988). Calculus for a new century: A pump, not a filter. Washington: The Mathematical Association of America.

Thompson, P. W. (2011). Quantitative reasoning and mathematical modeling. In S. Chamberlin, L. L. Hatfield, \& S. Belbase (Eds.), New perspectives and directions for collaborative research in mathematics education: Papers from a planning conference for WISDOMê. Laramie: University of Wyoming.

Thompson, P. W. (2013). In the absence of meaning. In K. Leatham (Ed.), Vital directions for research in mathematics education (pp. 57-93). New York: Springer.

Thompson, P. W., Castillo-Chavez, C., Culbertson, R. J., Flores, A., Greely, R., Haag, S., et al. (2007). Failing the future: Problems of persistence and retention in science, technology, engineering, and mathematics majors at Arizona State University. Tempe: Office of the Provost. 